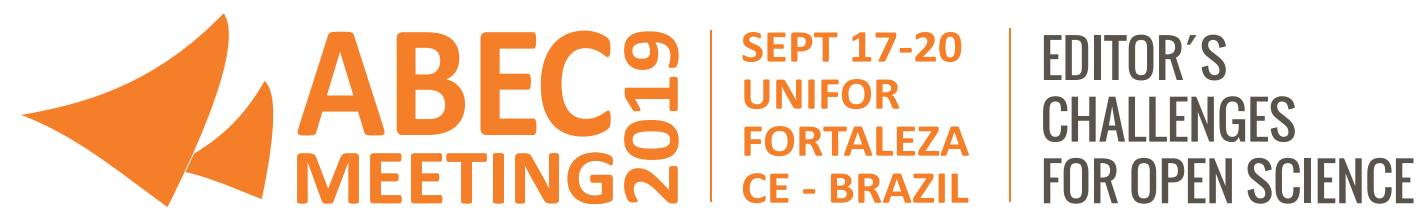

\title{
OS DESAFIOS ENFRENTADOS PELA EQUIPE EDITORIAL DO THE JOURNAL OF VENO- MOUS ANIMALS AND TOXINS INCLUDING TROPICAL DISEASES NA TRANSIÇÃO ENTRE DOIS PUBLISHERS DE RENOME INTERNACIONAL
}

The challenges faced by the Fournal of Venomous Animals and Toxins Including Tropical Diseases editorial team in the transition between two publishers of international rename

\section{Claudia Vilalva Cassaro}

Centro de Estudos de Venenos e Animais Peçonhentos. Journal of Venomous Animals and Toxins including Tropical Diseases

\section{Juliana Siani Simionato}

Journal of Venomous Animals and Toxins including Tropical Diseases

\section{Selma de Lourdes Pires Martins}

Journal of Venomous Animals and Toxins including Tropical Diseases

José Claudio Pires Martins

Journal of Venomous Animals and Toxins including Tropical Diseases

\section{Lucilene Delazari dos Santos}

Centro de Estudos de Venenos e Animais Peçonhentos. Journal of Venomous Animals and Toxins including Tropical Diseases

\section{Rui Seabra Ferreira Junior}

Centro de Estudos de Venenos e Animais Peçonhentos. Journal of Venomous Animals and Toxins including Tropical Diseases

\section{Benedito Barraviera}

Centro de Estudos de Venenos e Animais Peçonhentos. Journal of Venomous Animals and Toxins including Tropical Diseases

\section{COMO CITAR}

CASSARO, Claudia Vilalva et al. Os desafios enfrentados pela equipe editorial do The Journal of Venomous Animals and Toxins including Tropical Diseases na transição entre dois publishers de renome internacional. In: ABEC MEETING, 2019, Fortaleza. Anais... São Paulo: Associação Brasileira de Editores Científicos, 2019. http://dx.doi.org/10.21452/abecmeeting. 2019.202 


\section{RESUMO}

The Journal of Venomous Animals and Toxins nascido em 1995 dentro de uma Universidade pública, foi a primeira revista digital brasileira. Em 1998 foi selecionada para integrar a base SciELO (Scientific Electronic Library Online). Para se tornar mais abrangente em 2003 as Doenças Tropicais foram incluídas no seu escopo. Denominou-se a partir de então The Journal of Venomous Animals and Toxins including Tropical Diseases. Em 2006 foi selecionada para integrar duas Bases internacionais estratégicas: a Web of Science da Clarivate Analytics, que publica anualmente o Fator de Impacto, e a Scopus da Elsevier, que publica o Cites per Doc e o CiteScore. Como estes índices se estabilizaram até 2012 entre 0.30 e 0.50 o Conselho Editorial decidiu estabelecer parceria com a BioMed Central Springer-Nature, um publisher comercial de acesso aberto e renome internacional. Houve nesta ocasião uma reestruturação importante do periódico, incluindo a publicação em fluxo contínuo e a indexação no PubMed Central, com vistas à melhoria da qualidade e do aumento das métricas. Em 2018 o Fator de Impacto alcança o valor de 2.935, o Cites per Doc o de 2.973 e o CiteScore o de 2.630. Em seis anos os indicadores tiveram um incremento substancial, ou seja, de 0.5 para 2.9. Tendo em vista os elevados investimentos necessários para renovação da parceria, e por tratar-se de uma publicação acadêmica e não-comercial, o corpo editorial decidiu priorizar a manutenção do acesso aberto e garantir a sustentabilidade financeira do periódico, trazendo a publicação de volta ao Brasil a partir de 2019. A equipe SciELO decidiu apoiar esta iniciativa corajosa e tornou-se o novo parceiro neste desafio. As estratégias adotadas na transição estão descritas em detalhes no "relato de experiência".

\section{PALAVRAS-CHAVE}

Periódicos científicos eletrônicos; publisher; acesso aberto; sustentabilidade.

\section{ABSTRACT}

The Journal of Venomous Animals and Toxins, born in 1995 in a public university, was the first Brazilian digital publication. In 1998 it was selected to be part of the SciELO (Scientific Electronic Library Online) database and in 2003, in order to become more comprehensive, Tropical Diseases were included in its scope. It was then named The Journal of Venomous Animals and Toxins including Tropical Diseases (JVATiTD). In 2006, the Journal was selected to be part of two strategic international bases: Web of Science from Clarivate Analytics, which annually publishes the Impact Factor, and Scopus from Elsevier, which publishes Cites / Doc and CiteScore. As these indexes stabilized until 2012 between 0.30 and 0.50, the Editorial Board decided to firm a partnership with BioMed Central Springer-Nature, an internationally renowned open access publisher. A major restructuring of the journal occured on this occasion, including adopting a continuous publication model and indexing in PubMed Central, in order to improving quality and increasing metrics. In 2018 the FI reaches 2,935, Cites per doc 2,973 and CiteScore 2,630. In six years the indicators increased substantially, that is, from 0.5 to 2.9. Given the high investments needed to renew the partnership, the Editorial Board decided to prioritize the maintenance of open access and ensure the financial sustainability of the journal, bringing the publication back to Brazil from 2019. The SciELO team decided to support this courageous initiative and became the new partner in this challenge. The strategies adopted in the transition are described in detail in this experience report. 


\section{KEYWORDS}

Electronic scientific journals; publisher; open access; financial sustainability.

\section{INTRODUÇÃO}

Os periódicos ou revistas científicas são publicações em séries, divididas em partes ou fascículos numerados progressiva e cronologicamente e publicadas sob intervalos regulares e sem previsão de término acerca de resultados de pesquisas ou revisões de literatura. Tais produções são sempre assinadas por um ou mais autores e avaliadas por um ou mais editores ou pares (FACHIN; HILLESHEIM, 2006; GARRIDO; RODRIGUES, 2010; STUMPF,1998) e desempenham papel crucial na divulgação da informação em ciência (STUMPF, 1996; MIRANDA, PEREIRA, 1996)

Por muitas décadas, as contribuições científicas eram publicadas em meio impresso, no formato de revistas, livros ou até mesmo folhetos (MEADOWS, 2001). Com a popularização da internet, todos os trâmites da publicação, bem como esta, passaram a ocorrer também de forma eletrônica (NAZARÉ et al., 2017). Desde a década de 1970, surgiram esforços para informatizar o processo de editoração científica (STUMPF, 1996). Entretanto, a difusão do formato digital ocorreu somente nos anos 90 , onde o acesso aos artigos publicados por computadores domésticos foi se tornando mais comum (STUMPF, 1996). Tal iniciativa tornou a distribuição da produção científica mais rápida e simples (STUMPF,1998).

No Brasil, as primeiras publicações científicas datam do século XIX, e já surgiram com o objetivo de difundir o conhecimento divulgados em outros países. Neste momento, os jornais eram cotidianos e não-especializados. 'O Patriota', de 1813, é considerado o primeiro jornal a tratar de "ciência" (FREITAS, 2006). Já em 1956, o Instituto Brasileiro de Bibliografia e Documentação lança o "Periódicos Brasileiros de Cultura" (STUMPF,1998).

\section{EVOLUÇÃO DO JOURNAL OF VENOMOUS ANIMALS AND TOXINS INCLUDING TROPICAL DISEASES: DA CRIAÇÃO À ÚLTIMA TRANSIÇÃO}

Em 1995, o Centro de Estudos de Venenos e Animais Peçonhentos (CEVAP UNESP) lançou o Journal of Venomous Animals and Toxins (JVAT) (BARRAVIERA, 2015), a primeira revista científica eletrônica brasileira. Em seus primeiros anos, o JVAT tinha periodicidade semestral e sua divulgação ocorreu por meio de disquetes até 1999; e por CD-ROMs no período de 2000 a 2002. A partir de 2003, tanto o processo de submissão quanto o acesso aos artigos publicados passaram a ser totalmente digital (MARTINS et al., 2018). Também neste ano, a temática. Doenças Tropicais também foi inserida no escopo da revista, a qual passou a denominar-se The Journal of Venomous Animals and Toxins including Tropical Diseases (JVATiTD).

Em 2013, a equipe do JVATiTD firmou parceria com a Biomed Central Springer-Nature, um publisher internacional, a fim de expandir suas indexações e manter o acesso aberto (MARTINS et al., 2018; BARRAVIERA, 2013), reafirmando seu compromisso com a divulgação científica. No ano seguinte, foi conquistada a indexação nas bases Pubmed e Pubmed Central (SIMIONATO et al., 2018).

Entretanto, no ano de 2018, o corpo editorial da revista percebeu que os elevados investimentos requeridos pela Biomed Central inviabilizavam a autossuficiência da revista. A partir disso, 
o desligamento desta foi solicitado. Partiu-se, então, em busca de um novo parceiro para a empreitada de garantir a sustentabilidade financeira da revista.

A Scientific Library Online (SciELO) é uma biblioteca digital de revistas científicas brasileiras em formato eletrônico (PACKER, 1998), da qual o JVATiTD faz parte da coleção desde 1998. Tal instituição aceitou o desafio de abrigar nossa revista, atuando também como publisher.

Em parceria com o Scholar One Manuscripts, o SciELO disponibiliza um sistema de fluxo editorial, por meio do qual é feita a submissão e gestão de arquivos referentes a manuscritos. Tal sistema foi adotado com sucesso pelo corpo editorial e autores do JVATiTD nos primeiros meses de 2019.

A seguir, elencamos os principais desafios enfrentados pela equipe do JVATiTD e SciELO nestra transição:

- Desenvolvimento de um website próprio: Além da homepage do JVATiTD já existente no SciELO (Figura 1), optou-se por criar um novo website independente, mais abrangente e customizável para adequar-se ao dinamismo do periódico (Figura 2). Tal projeto foi finalizado com sucesso e, entre outros, possui link para a submissão de manuscritos, que é realizada por meio do Scholar One Manuscripts (mc04.manuscriptcentral.com/jvatitd-scielo).

Figura 1. Biblioteca do Journal of Venomous Animals and Toxins including Tropical Diseases no SciELO
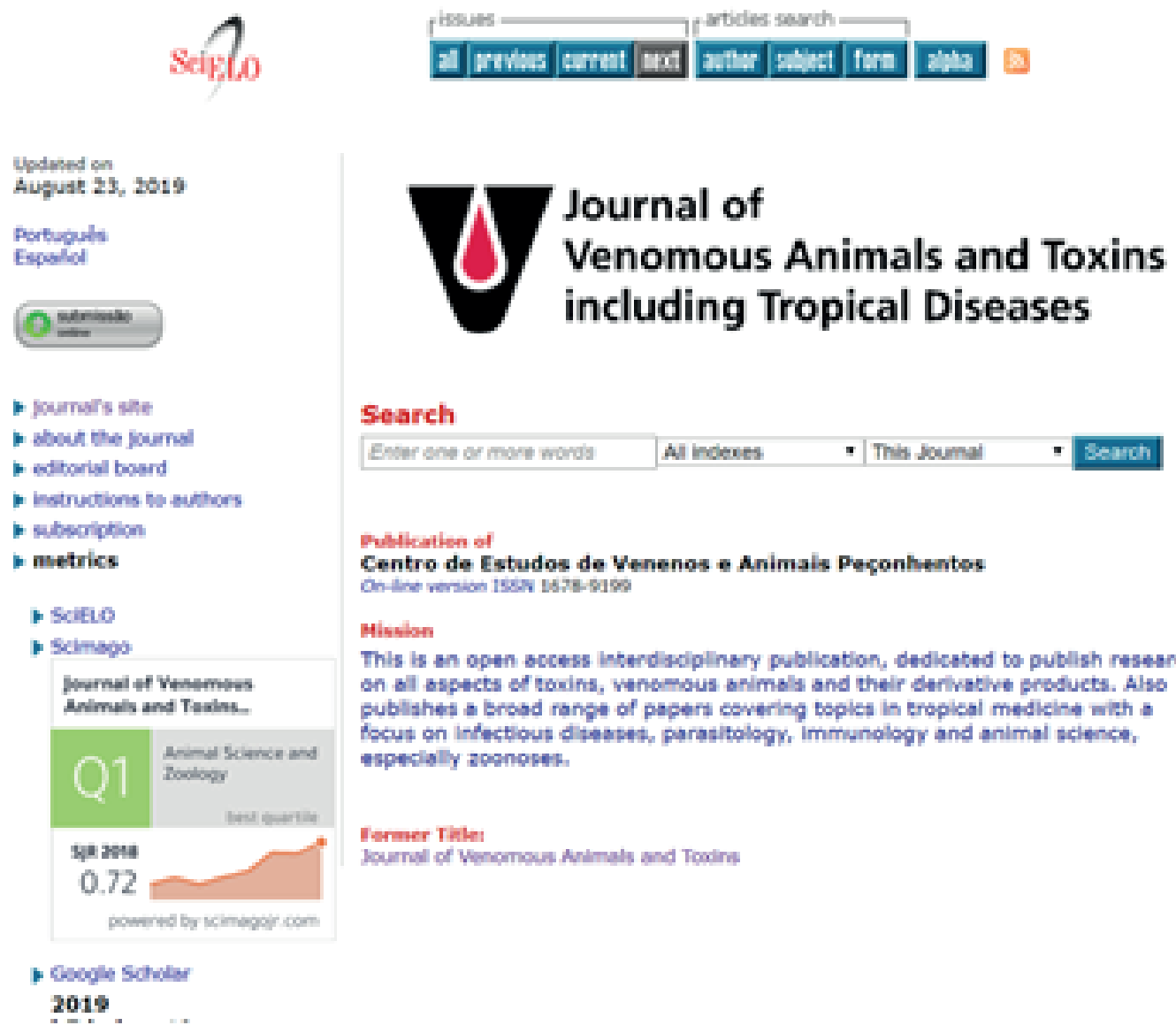

Dublicatien of

Centro de Estudos de Venenos a Animais Pegonhentes

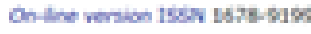

Mission

This is an open access interdisciplinery publication, dedicated to publish research on all aspects ef towins, venemous brimals and their derivative preducts. Also publishes a brobd range of papert covering toples is tropical medicine with a fecus on infectlous disebses, parasitology, immunology and animal science. especially aognoser.

Vermer Tille:

Journal of Venomous Animals and Toalins

Fonte: Captura de tela, 2019. 
Figura 2. Homepage do Journal of Venomous Animals and Toxins including Tropical Diseases

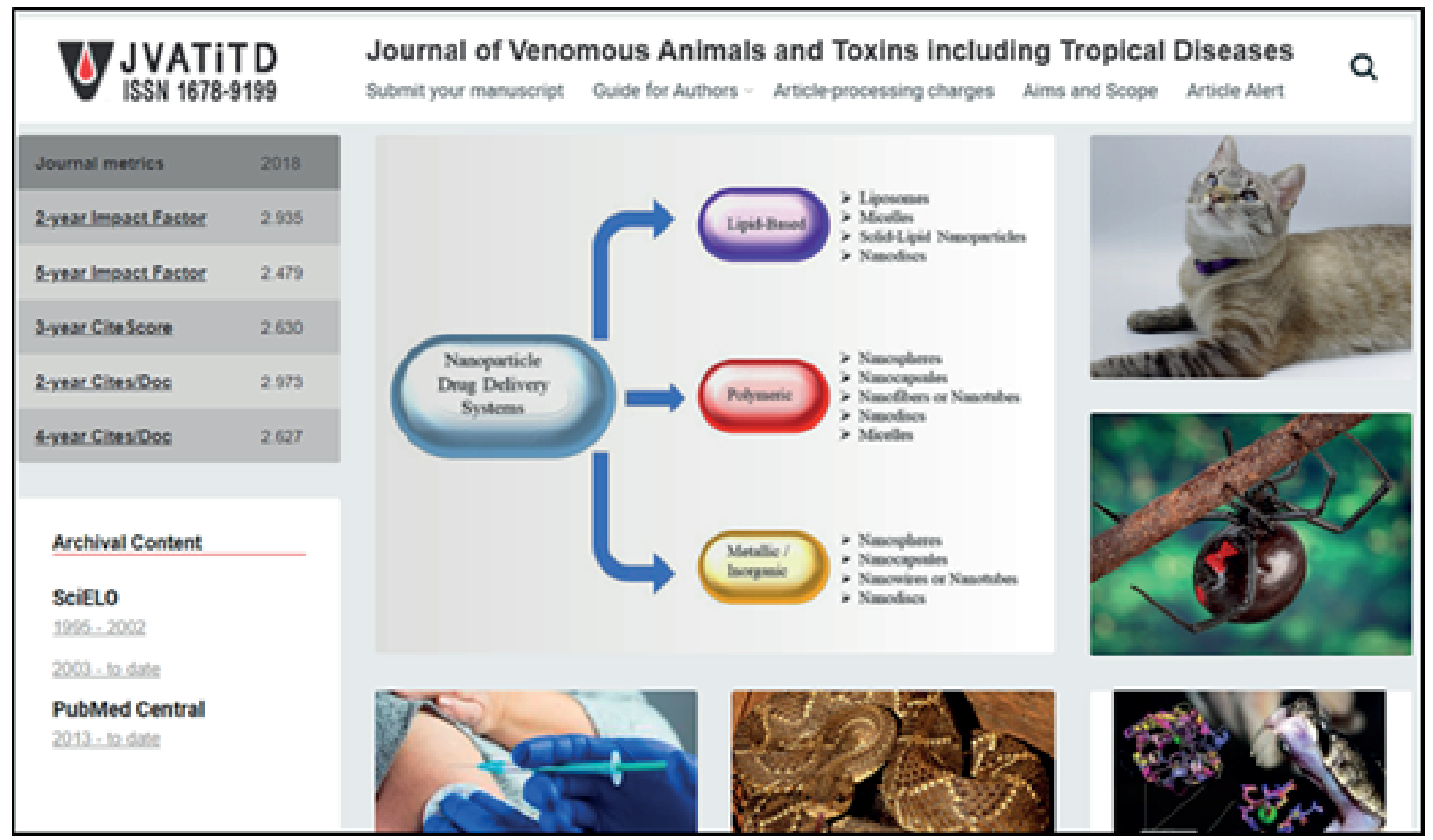

Fonte: Captura de tela, 2019.

- Transferência do domínio do site e unificação de domínios: Até o momento da transição, o site do JVATiTD estava vinculado à Biomed Central, a qual gentilmente auxiliou na transferência do domínio. Uma página referente à revista ainda está disponível (Figura 3), a qual possui arquivo com todos os artigos publicados na vigência da parceria. Aproveitou-se o momento para unificar os domínios ".org",."org.br" e ".com.br", a fim de facilitar o acesso de autores e pesquisadores à nova homepage.

Figura 3. Homepage do Journal of Venomous Animals and Toxins including Tropical Diseases mantido na Biomed Central, com acesso a todos os artigos publicados no período da parceria.

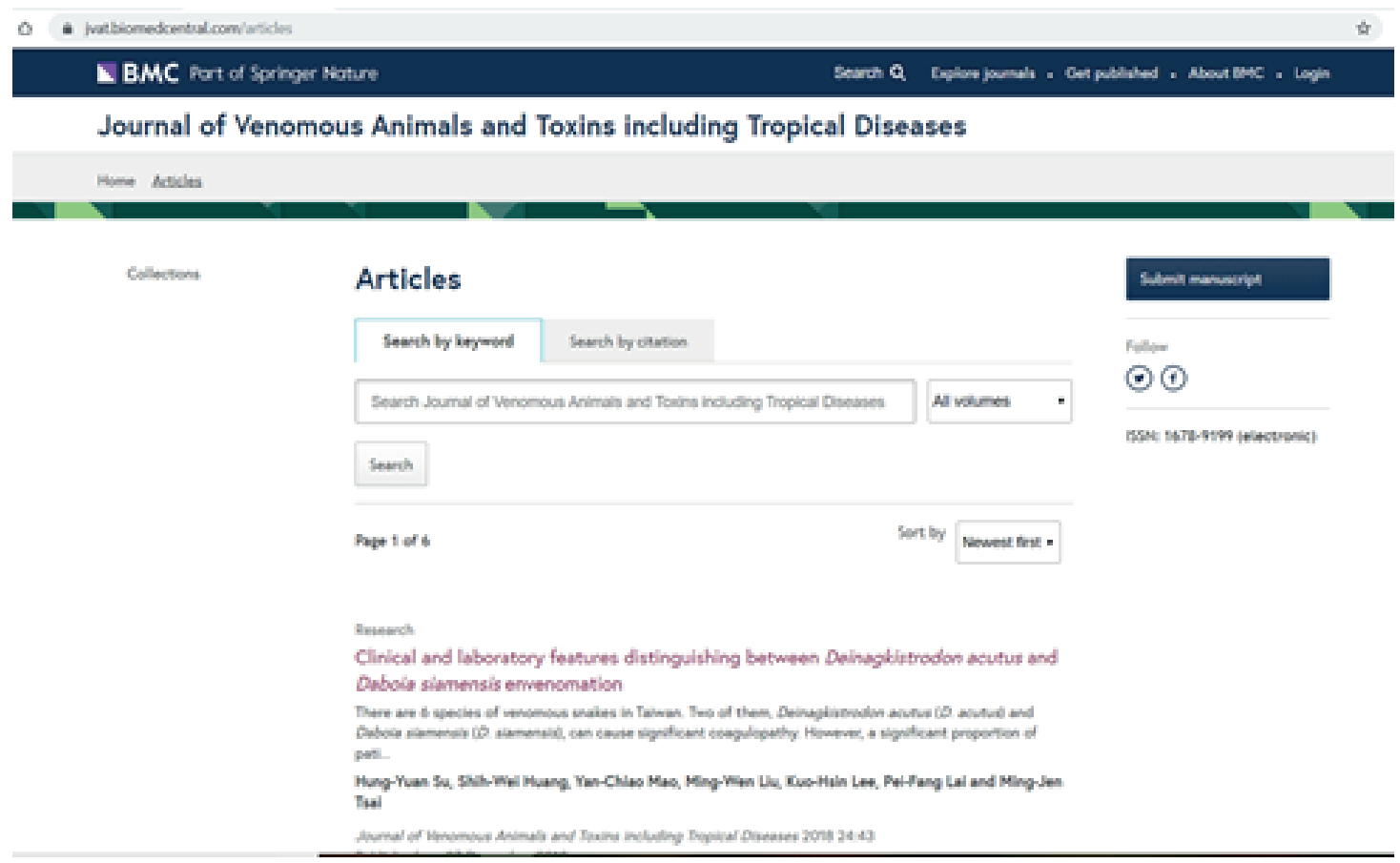

Fonte: Captura de tela, 2019. 
- Troca do DOI number: O DOI number é reconhecidamente um importante critério para identificação de arquivos científicos digitais. Dessa forma, foi necessária a tramitação por parte do SciELO para ser o novo DOI provider.

- Capacitação da equipe para a utilização do novo sistema editorial: A ocasião da transição de publisher representou o primeiro contato da equipe editorial do JVATiTD com o sistema Scholar One. Entretanto, todas as dificuldades foram contornadas pela capacitação oferecida pelo SciELO, bem como o suporte constante da equipe de gestão editorial do mesmo.

- Definição de novos guidelines para a submissão de manuscritos: Anteriormente, o JVATiTD seguia os guidelines definidos pela Biomed Central. Com a nova autonomia adquirida, o corpo editorial debruçou-se sobre as necessidades e formatos requeridos pela revista, delineando novas normas que atendam ao formato da publicação, adaptados a partir do padrão mundial vigen

A fim de priorizar a missão de acesso aberto à ciência, optou-se por tornar o JVATiTD um jornal não comercial, ou seja, sem fins lucrativos. As taxas de submissão foram reavaliadas de 2.000 para 500 dólares para autores estrangeiros e 300 dólares para autores nacionais, em uma clara ação de valorização e incentivo à produção científica nacional. Esta decisão deverá estimular os autores a escolher o JVATiTD para publicar e otimizar recursos financeiros. Optou-se, portanto, pela sustentabilidade financeira e autonomia editorial da revista.

As ações adotadas foram satisfatórias e hoje todos os percalços referentes à transição foram superados. Destacamos que nenhuma das indexações do JVATiTD foi perdida, em especial a da Pubmed Central (Figura 4), que garante grande visibilidade ao periódico. Por meio de ações inovadoras, os indicadores bibliométricos do JVATiTD vêm apresentando grande ascensão, atingindo Fator de impacto (Journal Citation Reports) de 2.935, CiteScore (Scopus) de 2.630 eCites per doc (Scimago Journal \& Country Rank) de 2.973 (Figura 5).

Figura 4. Indexação do Journal of Venomous Animals and Toxins including Tropical Diseases na Pubmed Central.

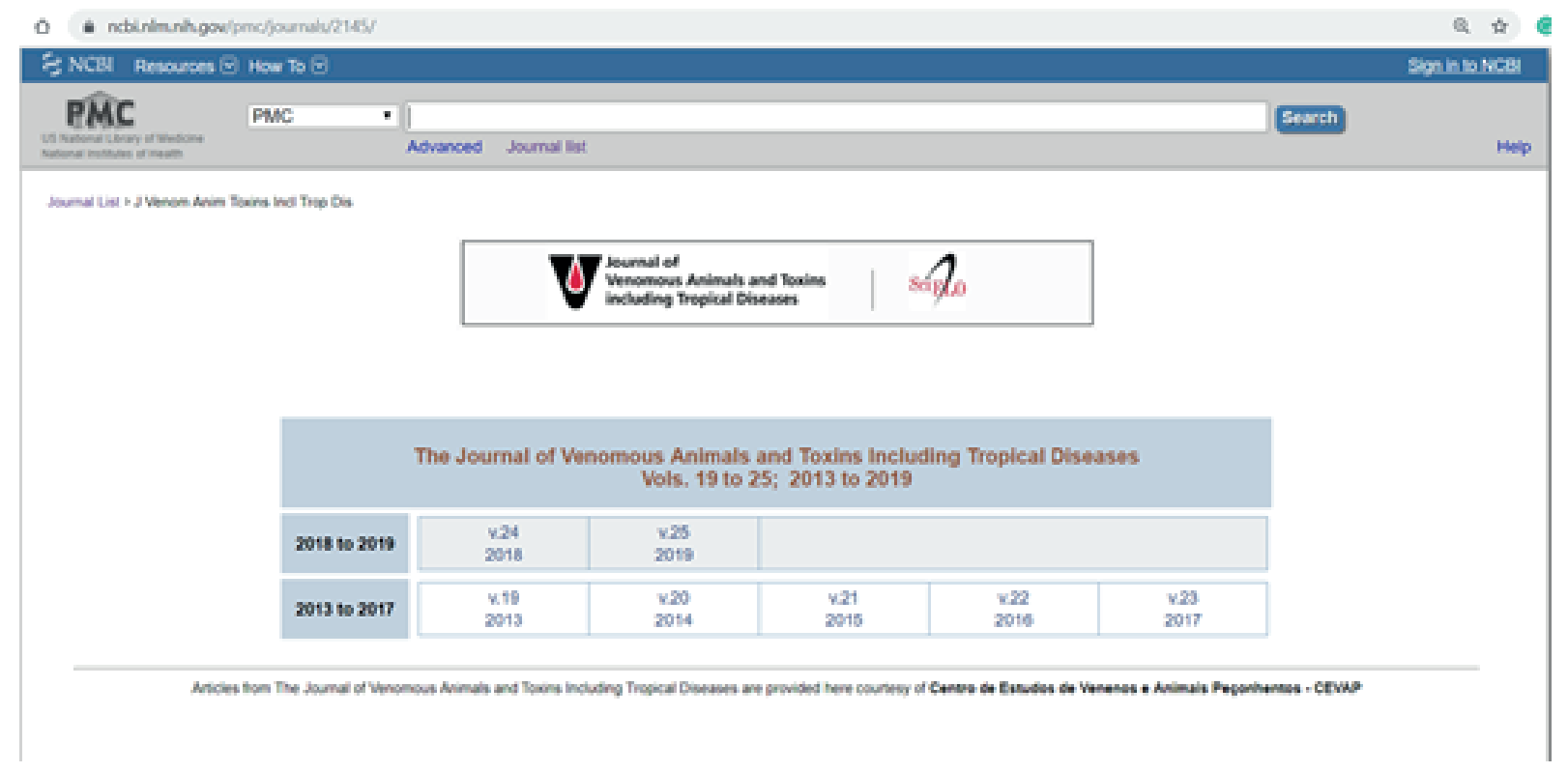

Fonte: Captura de tela, 2019. 


\section{REFERÊNCIAS}

BARRAVIERA, Benedito. CEVAP journal towards a new phase. Journal of Venomous Animals And Toxins Including Tropical Diseases, [s.I.], v. 19, n. 1, 2013.

BARRAVIERA, Benedito. CEVAP Journal: the first Brazilian electronic scientific publication turns 20 years old. Journal Of Venomous Animals And Toxins Including Tropical Diseases, [s.I.], V. 21, n. 1, dez. 2015.

FACHIN, Gleisy Regina Bories; HILLESHEIM, Araci Isaltina de Andrade, Periódico Científico: padronização e organização. Florianópolis: Ed. da UFSC, 2006.

FREITAS, Maria Helena. Considerações acerca dos primeiros periódicos científicos brasileiros. Ciência da Informação, v. 35, n. 3, p. 54-66, set/dez 2016.

GARRIDO, Isadora dos Santos; RODRIGUES, Rosangela Schwarz. Portais de periódicos científicos online: organização institucional das publicações. Perspectivas em Ciência da Informação, v.15, n.2, p.56-72, mai/ago. 2010.

MARTINS, Selma de Lourdes Pires et al. Do disquete às nuvens: A saga da primeira revista eletrônica científica brasileira. Ciência da Informação em Revista. v. 5, n.esp., p. 86-100, 2018.

MEADOWS, Jack. Os periódicos científicos e a transição do meio impresso para o eletrônico. Revista de Biblioteconomia de Brasília, v. 25, n.1, p. 5-14, 2001.

MIRANDA, Delly Bezerra; PEREIRA, Maria Nazaré de Freitas. O periódico científico como veículo de comunicação: uma revisão de literatura. Ciência da Informação, v. 25, n. 3, 1998.

NAZARÉ, Erik André et al. O digital object identifier (DOI) em periódicos científicos eletrônicos de comunicação e informação. RDBCl: Revista Digital Biblioteconomia e Ciência da Informação, v. 15, n. 3, p. 533-549, set/dez 2017.

PACKER, Abel Laerte et al. SciELO: uma metodologia para publicação eletrônica. Ciência da Informação, v. 27, n. 2, 1998.

SIMIONATO, Juliana Sciani et al. Como aumentar o fator de impacto nas bases Web of Science (WoS) e Scopus (Scimago): Ações implementadas pelo Journal of Venomous Animals and Toxins including Tropical Diseases. Ciência da Informação em Revista. v. 5, n. esp., p. 58-67, 2018.

STUMPF, Ida Regina Chitto. Passado e futuro das revistas científicas. Ciência da Informação, v. 25, n. 3, 1996.

STUMPF, Ida Regina Chitto. Reflexões sobre as revistas brasileiras. Intertexto, v. 1, n.3, p. 1-10, 1998. 
Por meio de ações inovadoras, os indicadores bibliométricos do JVATiTD vêm apresentando grande ascensão, atingindo Fator de impacto (Journal Citation Reports) de 2.935, CiteScore (Scopus) de 2.630 eCites per doc (Scimago Journal \& Country Rank) de 2.973 (Figura 5).

Figura 5. Ascenção das métricas do Journal of Venomous Animals and Toxins including Tropical Diseases.

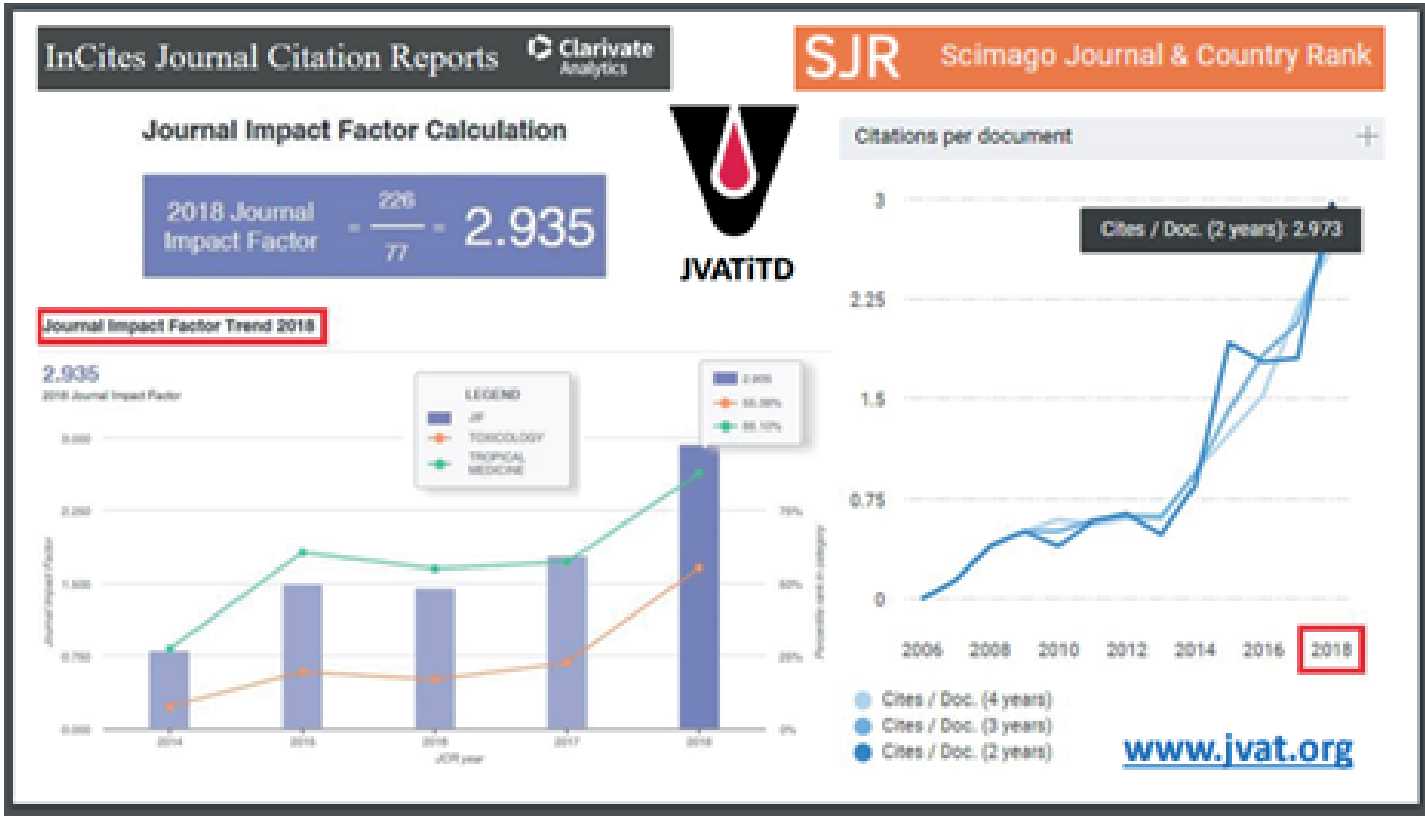

Fonte: Captura de tela, 2019.

\section{CONSIDERAÇÕES FINAIS}

A decisão de transição de publisher mostra o pioneirismo do JVATiTD frente à resolução de problemas, que buscou novas alternativas diante da impossibilidade de renovação de parceria internacional por recursos financeiros. A parceria de seis anos junto à Biomed Central proporcionou grande aprendizado ao corpo editorial da revista, produtividade e visibilidade ao periódico e foi imprescindível para o significativo aumento do fator de impacto e demais indicadores da mesma. Em busca de sua sustentabilidade, uma nova parceria junto ao SciELO foi firmada, e espera-se que a visibilidade e crescimento dos indicadores bibliométricos de tal publicação científica, que se reinventa com dinamismo e inovação há mais de duas décadas, sejam mantidos e até mesmo otimizados.

\section{AGRADECIMENTOS}

A equipe editorial do JVATiTD agradece à Biomed Central - Springer Nature pelos anos de parceria, bem como pelo profissionalismo e solicitude em todo o processo de transição, em especial à Dana Berry, Linda van der Wende, Laurence Cheeseman e Mariana Biojone Brandão. Agradece, também, à toda equipe de gestão editorial do SciELO e colaboradores pela capacitação e suporte, em especial ao Alex Mendonça, Carolina Tanigushi e Francine Curivil, Solange Santos e Abel Packer. 